\title{
Performance Analysis of Peak-to-Average Power Ratio Reduction Techniques for Wireless Communication Using OFDM Signals
}

\author{
Pawan Sharma1, Seema Verma ${ }^{2}$ \\ ${ }^{1}$ Department of Electronics and Communication, GGSIP University, \\ Bhagwan Parshuram Institute of Technology, Delhi-110089, India \\ 2 Department of Electronics, Banasthali University \\ Rajasthan-304022, India
}

\begin{abstract}
Orthogonal Frequency Division Multiplexing (OFDM) has been currently under intense research for broadband wireless transmission due to its robustness against multipath fading. However OFDM signals have a problem with high Peak-toAverage power ratio (PAPR) and thus, a power amplifier must be carefully manufactured to have a linear input-output characteristic or to have a large input power back-off. In this paper, some of the important PAPR reduction techniques which have been compared based on computational complexity, bandwidth expansion, spectral spillage and performance.
\end{abstract}

Keywords: Orthogonal Frequency Division Multiplexing (OFDM), Peak-to-Average Power Ratio (PAPR), Complementary Cummulative Distribution Function (CCDF), High Power Amplifier (HPA), Partial Transmit Sequence (PTS), Selected Mapping (SLM).

\section{Introduction}

The growth of mobile wireless communications has been producing the demand for high-speed, efficient and reliable communication over the hostile wireless medium. As a modulation scheme for such applications, Orthogonal Frequency Division Multiplexing (OFDM) possesses several desirable attributes, such as immunity to the inter-symbol interference, robustness with respect to multi-path fading, and ability for high data rates. Thus, OFDM has been proposed in various wireless communication standards such as IEEE802.11a standard for wireless Local Area Networks (WLAN), IEEE802.16a standard for Wireless Metropolitan Area Networks (WMAN), digital audio/video broadcasting, Terrestrial Digital Video Broadcasting (DVB-T), the ETS1 HIPERLAN/2 standard and high speed cellular data [1]. However, one of the major drawbacks of OFDM system has been its high Peak-to-Average Power Ratio (PAPR). The high
PAPR brings the OFDM signal distortion in the non-linear region of high power amplifier (HPA) and the signal distortion induces the degradation of bit error rate (BER). Moreover, to prevent spectral growth of the multicarrier signal in the form of intermodulation among subcarriers and out-of-band radiation, the transmit power amplifier has to be operated in its linear region [2]. If the HPA is not operated in linear region with large power back-offs, it is impossible to keep the out-ofband power below the specified limits. This situation leads to very inefficient amplification and expensive transmitters.

Therefore, it has been important and necessary to research on the characteristics of the PAPR, including its distribution and reduction, in OFDM systems, in order to utilize the technical features of the OFDM.

In 2005, the Wimedia alliance worked with the European Computer Manufactures Associations (ECMA) and announced the establishment of the WiMedia MB-OFDM (Multiband Orthogonal Frequency Division Multiplexing), UltraWideband (UWB) radio platform as their global UWB standard, ECMA-368 and the latest updated version incorporated spectral nulling. ECMA -368 was also chosen as the physical layer (PHY) for the high data rate wireless specifications, for high-speed wireless USB (W-USB), Bluetooth 3.0 and wireless high-definition Media Interface (HDMI) [3].

To reduce the PAPR several techniques have been proposed such as partial transmit sequences (PTS) [4, 5], selective mapping (SLM) [6,7], clipping [8,9] clipping and filtering [10], coding [11], tone reservation (TR) and tone injection (TI) [12]. Each of these methods has a different cost for the reduced PAPR.

Although some techniques of PAPR reduction have been summarized, it is still necessary to give a comprehensive review of PAPR reductions in terms of transmission power, data rate loss, implementation complexity and BER performance, etc. 


\section{OFDM SYSTEM MODEL}

In OFDM system, a block of $\mathrm{N}$ symbols, $X_{n}, n=1,2 \ldots N-1$, is formed with each symbol modulating one of a set of subcarriers, $\left\{f_{n}, \mathrm{n}=0,1,2 \ldots \mathrm{N}-1\right\}$, where $N$ is number of subcarriers. The $N$ subcarriers are chosen to be orthogonal, that is, $f_{n}=n \Delta f=n / T$, where $\mathrm{T}$ is the original symbol period. The OFDM baseband signal is denoted by:

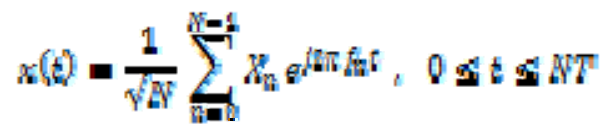

Where $\mathrm{j}=\sqrt{ }-1$.

A guard Interval is inserted to each OFDM symbol as a cyclic prefix. Finally, an OFDM signal stream is obtained after demultiplexing.

The PAPR of OFDM is defined as

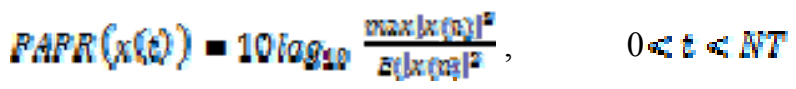

Where $E\{\quad\}$ is the expectation.

In principle, there has been more concern with reducing the PAPR of the continuous-time OFDM signals, since the cost and power dissipation of the analog components often dominates. However, most existing PAPR reduction methods can only be implemented on the discrete-time OFDM signals by IFFT. The IFFT output can be expressed as follow:

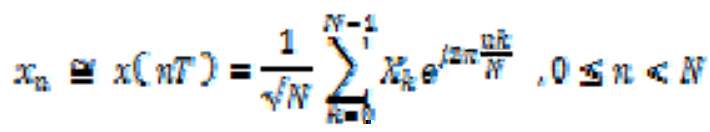

the corresponding PAPR can be defined as

$\operatorname{RARR}(x)=10 \log \frac{\max |x|^{2}}{E\left[x^{2}\right]}$

\subsection{Motivation Of PAPR Reduction}

Most radio systems employ the HPA in the transmitter to obtain sufficient transmission power. For the purpose of achieving the maximum output power efficiency, the HPA is usually operated at or near the saturation region. Moreover, the nonlinear characteristic of the HPA is very sensitive to the variation in signal amplitudes.

However, the variation of OFDM signal amplitudes is very wide with high PAPR. Therefore, HPA will introduce inter-modulation between the different subcarriers and introduce additional interference into the systems due to high PAPR of OFDM signal. This additional interference leads to an increase in BER. In order to lessen the signal distortion and keep a low BER, it requires a linear work in its linear amplifier region with a large dynamic range. However, this linear amplifier has poor efficiency and is so expensive. Power efficiency is very necessary in wireless communication as it provides adequate area coverage, saves power consumption and allows small size terminals etc. It has been therefore important to aim at a power efficient operation of the non-linear HPA with low back-off values and try to provide possible solutions to the interference problem brought about. Hence, a better solution has been to try and prevent the occurrence of such interference by reducing the PAPR of the transmitted signal with some manipulations of the OFDM signal itself.

Large PAPR also demands the Digital-to-Analog Converter (DAC) with enough dynamic range to accommodate the large peaks of the OFDM signals. Although, a high precision DAC supports high PAPR with a reasonable amount of quantization noise, but it might be very expensive for a given sampling rate of the system. Whereas, a lowprecision DAC would be cheaper, but its quantization noise would be significant, resulting in reduction of the Signal-toNoise Ratio (SNR) when the dynamic range of DAC is increased to support high PAPR. Furthermore, OFDM signals show Gaussian distribution for large number of subcarriers, which means the peak signal rarely occur and uniform quantization by the ADCs is not desirable. If clipped, it will introduce in-band distortion and out-of-band radiation (adjacent channel interference) into the communication systems. Therefore, the best solution has been to reduce the PAPR before OFDM signals have been transmitted into nonlinear HPA and DAC.

\section{LATEST PAPR REDUCTION TECHNIQUES IN OFDM SYSTEMS}

In this section, various techniques for reducing the PAPR have been discussed .

\subsection{Clipping And Filtering}

The OFDM signal contains high peaks so Clipping And Filtering (CAF) is used in this system [13]. The generated OFDM signal is transferred to the clipping block. In the clipping part, when amplitude exceeds a threshold, the amplitude is hard-clipped while the phase is saved. 
www.IJCSI.org

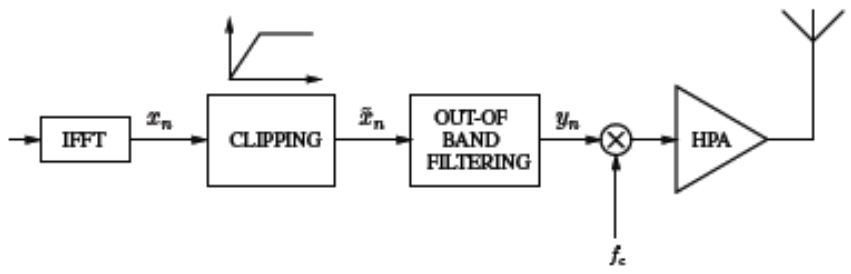

Fig.1. The OFDM transmitter including clipping scheme.

Namely, when we assume a phase of baseband OFDM signal $x_{n}$ is $\Phi_{n}$ and the threshold is A, the output signal after clipping is shown as

$$
x_{\mathrm{n}}= \begin{cases}A e^{\beta A n} & \left(\left|x_{n}\right| \geq A\right) \\ x_{n} & \left(\left|x_{n}\right| \leq A\right)\end{cases}
$$

The clipping causes both in-band and out-of-band distortion because of non-linear operation of the clipping, The in-band distortion causes degradation of BER, while distortion also causes out-of-band emission. To decrease the interference to neighboring channels, out-of-band components must be reduced with a band-limiting filter. Due to the non-linearity, clipping operations causes out-of-band components. Suppressing the components by band-limiting filters makes clipped peak signal expand. When CAF is applied to an oversampled signal, the peak regrowth becomes comparatively small [14]. However, it has been inevitable that the PAR is larger than the clipping threshold. For these reasons, some peak regrowth reduction methods have been proposed. A straightforward way of peak growth reduction has been repeated CAF method. In this method, clipping and filtering are applied repeatedly, and peak regrowth is reduced gradually. In the paper it has been shown, that repeated clipping and filtering significantly reduces the PAR. The method is effective, however, the calculation cost increases proportionally to the number of repeats, and filtering delay also increases. So there has been a tradeoff between PAR and the system cost.

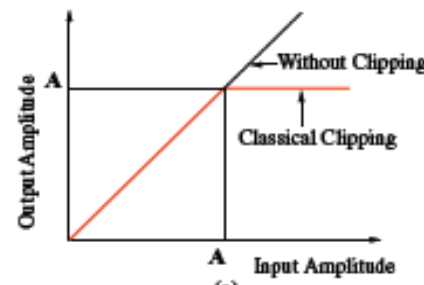

(a)

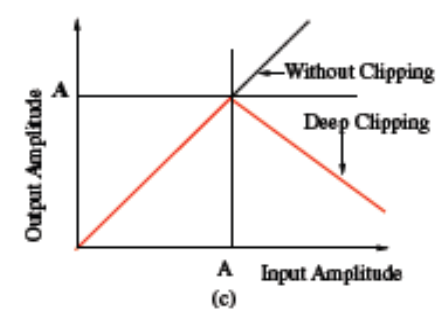

Fig.2. Functions-Based Clipping for PAPR Reduction
There have been four other clipping techniques: Classical Clipping (CC), Heavyside Clipping (HS), Deep Clipping (DC) and Smooth Clipping (SC) whose functions are depicted in Fig.2. [15]. Rewriting the discrete-time OFDM signal $x_{n}$ to polar coordinates gives $x_{n z}=r_{n} e^{j \phi n}$, where $r_{n}$ represents the amplitude of $x_{\mathrm{n}}$ and $\phi_{\mathrm{n}}$ represents the phase of $x_{n}$, The clipped signal $\overline{x_{n}}$ is expressed as

$\overline{x_{n}}=f\left(p_{n}\right) e^{* n}$

(6)

Where the clipping function $f(r)$ is expressed below according to the type of clipping used.

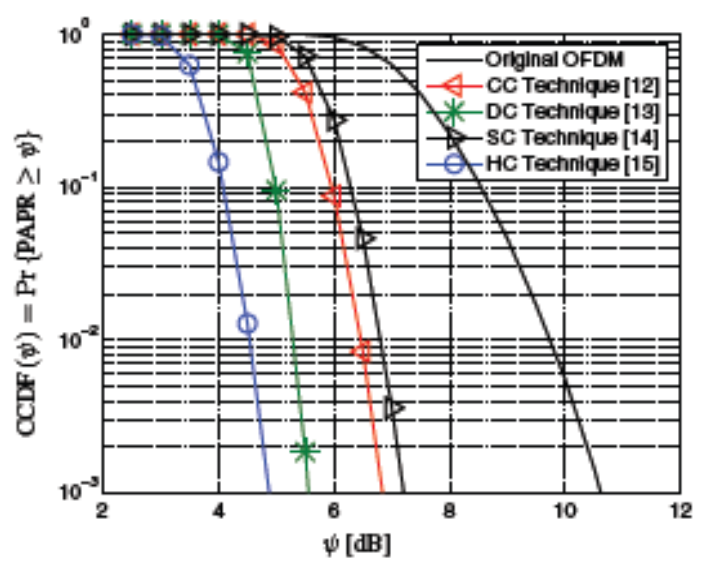

Fig.3. PAPR reduction performance of CC,HC,HC and DC techniques.

The Classical Clipping (CC) proposed in [16] has been one of the most popular clipping techniques for PAPR reduction known in the literature [11]. It is sometimes called hard clipping or soft clipping, to avoid any confusion; it is called Classical Clipping (CC) in this paper. The function-based clipping used for $\mathrm{CC}$ technique is defined below and depicted in Fig. 2(a).

$f(r)=\left\{\begin{array}{lll}r & r & \leq A \\ A & r & \cdots\end{array}\right)$, where $\mathrm{A}$ is the clipping level.

Heavyside Clipping, often called hard clipping has been used in [17] as a baseband nonlinear transformation technique to improve the overall communication system performance. The heavyside function is expressed below and depicted in Fig.2(b).

$$
f(r)=\mathrm{A}, \quad \forall r \geq 0
$$

Deep Clipping (DC) has been proposed in [14] to solve the peaks regrowth problem due to the out-of-band filtering. So, in DC technique, the clipping function has been modified in order to 'deeply' clip the high amplitude peaks. A parameter called clipping depth factor has been introduced in order to control 
www.IJCSI.org

the depth of the clipping. The function-based clipping used for DC technique is defined below and depicted in Fig.2

(c).
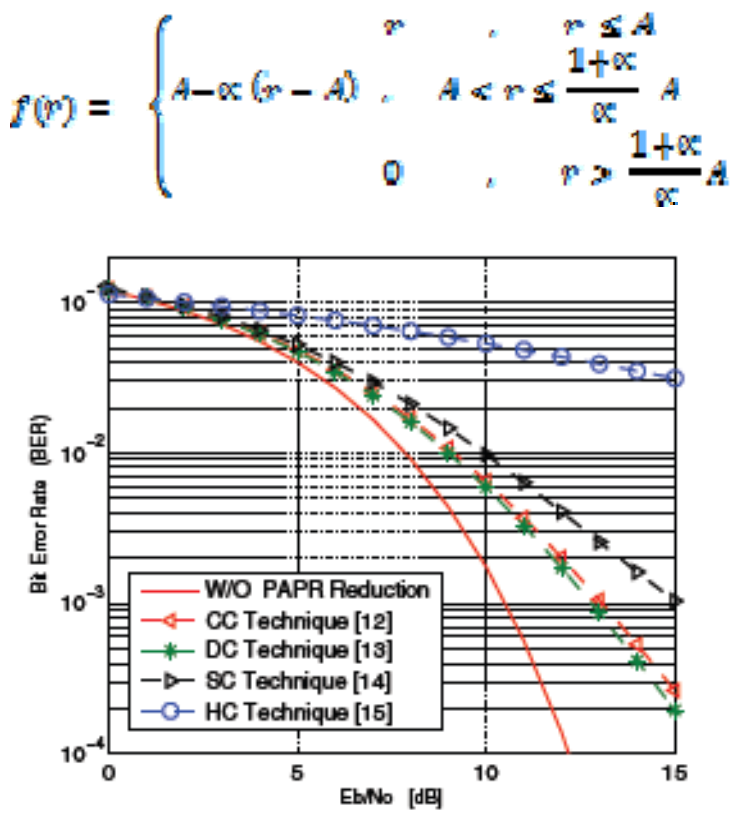

Fig.4. BER performance of CC,SC,HC and DC techniques.

The function for Smooth Clipping (SC) [18] has been defined below and depicted in Fig. 2(d )

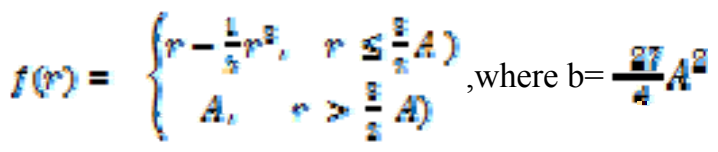

\subsection{Partial Transmit Sequences (PTS)}

For PAPR reduction using partial transmit sequence a typical OFDM system with input data block in $\mathrm{X}$ has been partitioned into $\mathrm{M}$ disjoint subblocks of clusters, which are represented by the vectors $\left\{\mathrm{X}^{(\mathrm{m})}, \mathrm{m}=0,1, \mathrm{M}-1\right\}$ [19]. Fig.5. shows the block diagram of the PTS technique .

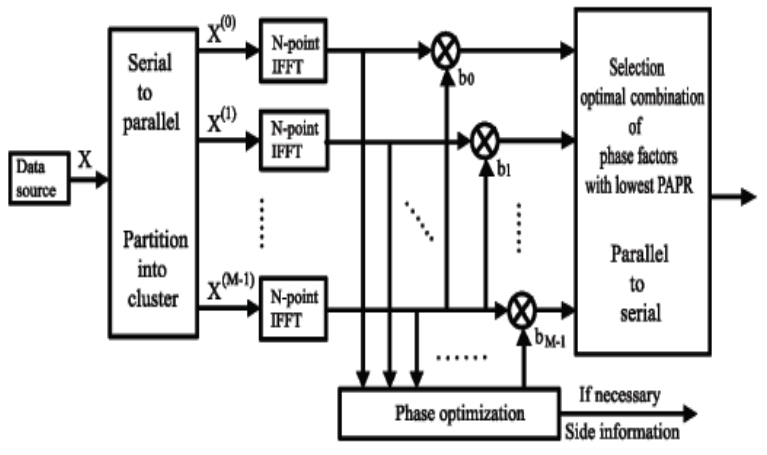

Fig.5. Block Diagram of PTS Technique

Therefore,

$$
X=\sum_{M=0}^{M-1} X^{(x)}
$$

Where $\quad \mathrm{X}^{(\mathrm{m})}=\left[\mathrm{X}_{0}{ }^{(\mathrm{m})} \quad \mathrm{X}_{1}^{(\mathrm{m})} \ldots \ldots . . \mathrm{X}_{\mathrm{N}-1}{ }^{(\mathrm{m})}\right] \quad$ with $X_{k}^{(m)}=X_{k}$ or $0(0 \leq m \leq M-1)$. In general, for PTS scheme, the known subblock partioning methods can be classified into three categories: adjacent partition, interleaved partition and pseudo-random partition.

Then, the subblocks $\mathrm{X}^{(\mathrm{m})}$ are transformed into $\mathrm{M}$ time-domain partial transmit sequences

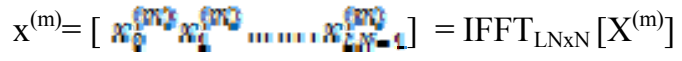

These partial sequences are independently rotated by phase factors $\mathbf{b}=\left\{\mathrm{b}_{\mathrm{m}}=\mathrm{e}^{\mathrm{j} \theta \mathrm{m}}, \mathrm{m}=0,1, \ldots . . \mathrm{M}-1\right\}$. The objective has been to optimally combine the M subblocks to obtain the time domain OFDM signals with the lowest PAPR

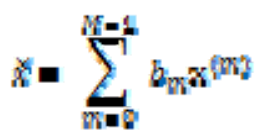

Assuming that there are $\mathrm{W}$ phase angles to be allowed, thus $\mathrm{b}_{\mathrm{m}}$ has the possibility of $\mathrm{W}$ different values. Therefore, there are $\mathrm{W}^{\mathrm{M}}$ alternative representations for an OFDM symbol.

The PTS technique significantly reduces the PAPR, but unfortunately, finding the optimal phase factors has been a highly complex problem. In order to reduce the search complexity, the selection of the phase factors has been limited to a set of finite number of elements. The Exhaustive Search Algorithm (ESA) [20] has been employed to find the best phase factor. However, the ESA requires an exhaustive search over all combinations of the allowed phase factors and has exponential search complexity with the number of subblocks. To reduce the computational complexity, some simplified search techniques have been proposed such as the Iterative Flipping Algorithm (IFA) [21]. Although the IFA significantly 
www.IJCSI.org

reduces the search complexity, there has been some gap between its PAPR reduction performance and that of the ESA. A Cross-Entropy (CE) based method has been proposed by Jung-Cheih Chen [20] for obtaining the optimal phase factors for the PTS technique to reduce the PAPR. Jung-Cheih Chen [22] has proposed a Quantum-Inspired Evolutionary Algorithm (QEA) based method to obtain the optimal phase factor for the PTS technique. Abolfazl et al.[23].has proposed an AutoCorrelation Function (ACF) to develop a new PTS subblocking technique using Error-Correcting Codes (ECCs). This technique minimizes the number of repeated subcarrier with a subblock and provides better PAPR reduction than pseudorandom or m-sequence subblocking.

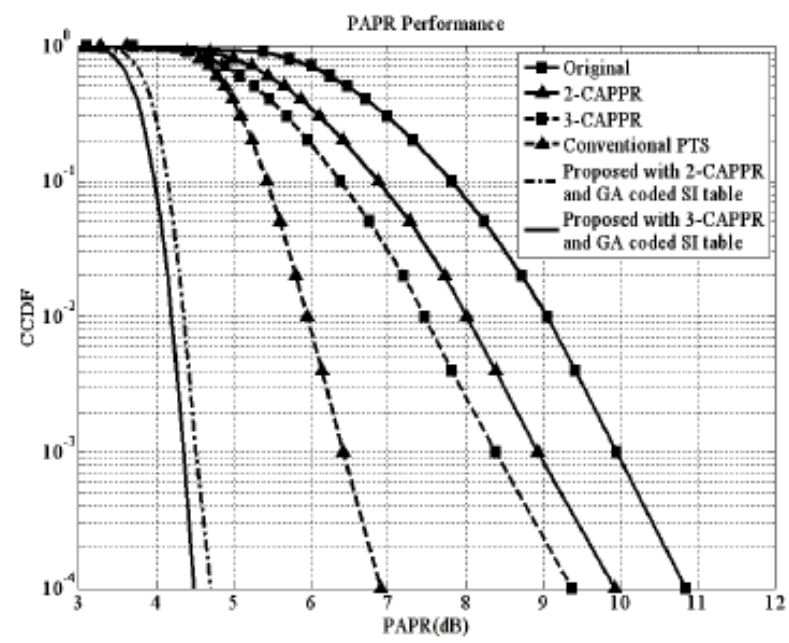

Fig.6.PAPR performance of an OFDM signal

\subsection{Selected Mapping Technique}

In SLM, the input data sequences have been multiplied by each of the phase sequences to generate alternative input symbol sequences. Each of the alternative input data sequences is made the IFFT operation, and then the one with the lowest PAPR is selected for transmission [11]. A block diagram of the SLM technique has been depicted in Fig.7. Each data block is multiplied by $\mathrm{V}$ different phase factors, each of length $\mathrm{N}, \mathbf{B}_{\mathbf{v}}=$ $\left[b_{v, 0}, b_{v, 1}, \ldots . b_{u, N-1}\right]^{T}(v=0,1, \ldots . . V-1)$, resulting in $\mathrm{V}$ different data blocks. Thus, the $\mathrm{v}^{\text {th }}$ phase sequence after multiplied is $\mathbf{X}^{v}=\left[\mathrm{X}_{0} \mathrm{~b}_{v, 0}, \mathrm{X}_{1} \mathrm{~b}_{v, 1}, \ldots, \quad \mathrm{X}_{\mathrm{N}-1} \mathrm{~b}_{v, \mathrm{~N}-1}\right]^{\mathrm{T}}(v=0,1, \ldots . \mathrm{V}-1)$. Therefore, OFDM signals becomes as

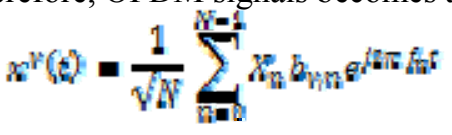

Where $0 \leq t \leq N T, v=1,2, \ldots \ldots, \mathrm{V}-1$.

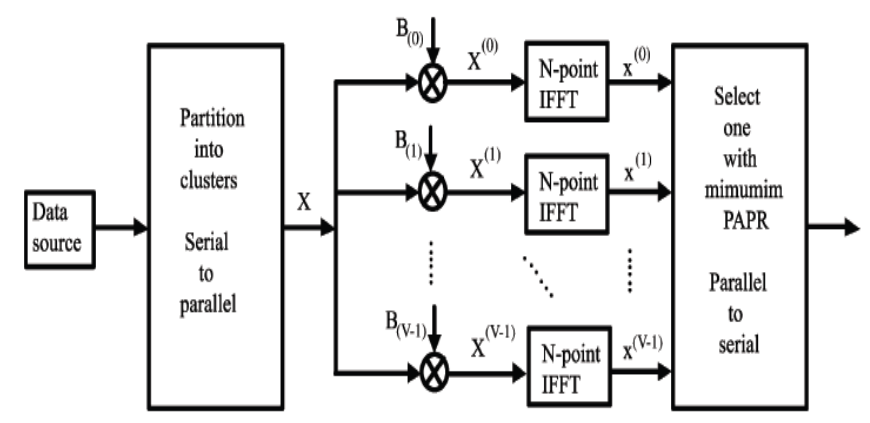

Fig.7. Block Diagram of SLM Technique

Among the data block $X^{v}(v=0,1, \ldots V-1)$, only one with the lowest PAPR has been selected for transmission and the corresponding selected phase factors $b_{v, n}$ also should be transmitted to receiver as side information. For implementation of SLM OFDM systems, the SLM technique needs V IFFT operation and the number of required bits as side information is $\left[\log _{2} \mathrm{~V}\right]$ for each data block. Therefore, the ability of PAPR reduction in SLM depends on the number of phase factors $\mathrm{V}$ and the design of the phase factors. Some extension of SLM also has been proposed to reduce the computational complexity and number of the bits for side information transmission [24].

In symbol scrambling techniques the input data sequence has been "scrambled" using a number of specialized scrambling sequences. The sequence which produces the lowest PAPR is the one used for transmission. Selected Mapping (SLM) has been one of the most popular signal scrambling techniques used to reduce the PAPR of OFDM signals. The SLM technique proposed by Bauml et al. [26] takes the OFDM subcarrier data block to be transmitted and multiples it element-wise by a number of phase adjustment vector sets. The new statistically independent phase adjusted OFDM frames represent the same transmitted information, but have different PAPR values. The OFDM frame that has the lowest PAPR is then selected to be transmitted. As reported in [26] the SLM technique can provide a $0.1 \%$ probability PAPR reduction of $2.8 \mathrm{~dB}$ (from $10.4 \mathrm{~dB}$ to $7.6 \mathrm{~dB}$ ) when applied to 128-subcarrier QPSK-OFDM symbols. However, one drawback of this technique is that some additional information relating to the phase vector set that produces the lowest PAPR also requires to be transmitted along with the OFDM signal. This extra information increases the overhead.

Breiling et al.[25] have proposed an SLM technique that avoids explicit use of side information by appending a label that lets the receiver identify the scrambled sequence which produces the lowest PAPR. This scrambler uses a number of shift-register stages with internal feedback paths resulting in error propagation in the receiver causing an increased reception bit-error-rate (BER). Various SLM techniques without side information have been proposed, e.g., Jayalath and Tellambura [27] have proposed a maximum likelihood (ML) decoder for SLM and Partial Transmit Sequences (PTS) techniques that requires no side information. The receiver exploits the large Hamming distances in the set of 
www.IJCSI.org

the phasing sequences and uses optimum hard decision for each subcarrier.

Stephane and Samer [28] have proposed a SLM technique without side information. This method takes into account the increase in average energy with PAPR reduction which is identical to that of classical SLM. A recursive Selected Mapping (RSLM) for PAPR reduction has been proposed by Lingyin and Yewen [29]. where the PAPR reduction has been a little better than that of SLM with high reduction in computational complexity. Xiaowen and Seungmin [30] have proposed a look-up table method (LUT) by making use of the feature that the PAPR performance is independent of modulation schemes in normal OFDM. This method shows the regulations of selective efficient phase rotation factors. It proves a way of achieving the most efficient PAPR performance for SLM-OFDM, but limited to FFT size 8.

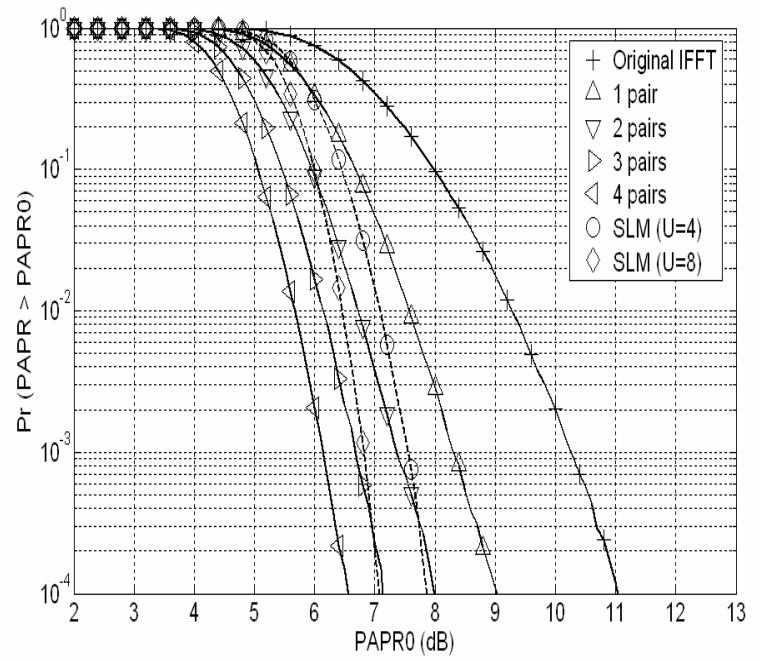

Fig.8. CCDF of the PAPR obtained with the SLM techniques.

\subsection{Tone Reservation and Tone Injection}

Tone Reservation (TR) and Tone Injection (TI) are two efficient techniques to reduce the PAPR of OFDM signals [11]. Fig.9. describes the block diagram of TR and TI, in which the key idea is that both transmitter and receiver reserve a subset of tones for generating PAPR reduction signals, $c$. Note that these tones are not used for data transmission.

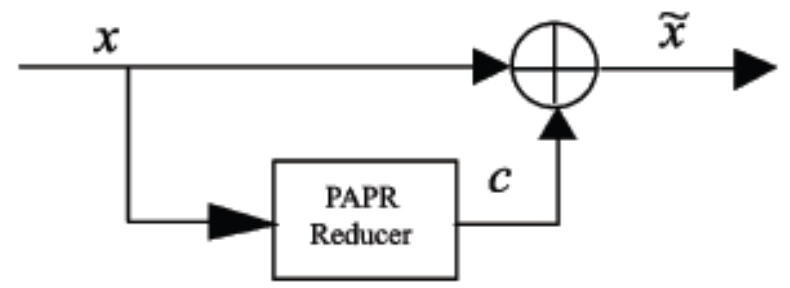

Fig.9. Block Diagram of TR/TI approaches for PAPR Reduction.
In TR, the objective has been to find the time domain signal $c$ to be added to the original time domain signal $x$ to reduce the PAPR. Let $\left\{\mathbf{c}=\sigma_{n} \mid n=0,1, \ldots \ldots, N-1\right\}$ denote complex symbols for tone reservation at reserved tones. Thus, the data vector changes to $\mathbf{x}+\mathbf{c}$ after tone reservation processing, and this results in a new modulated OFDM signals as

$$
\mathcal{Z}=\operatorname{IFFT}(\mathrm{x}+\mathrm{c})=\mathrm{X}+\mathrm{C}
$$

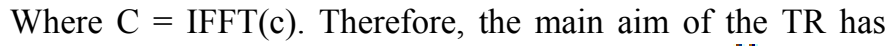
been to find out the proper $\mathbf{c}$ to make the vector $\tilde{\mathbf{X}}_{\text {with low }}$ PAPR. To find the value of $\mathbf{c}$, a convex optimization problem must be solved which can be easily cast as a linear programming problem.

Similarly, TI also uses an additive correction to optimize $\boldsymbol{C}$ in (15). The basic idea of TI has been to extend the constellation and thus the same data point corresponds to multiple possible constellation points. One option has been to replicate the original shaded constellation into several alternative ones. Therefore, $C$ has been a translation vector such that $\mathrm{C}=(\overline{\boldsymbol{X}})$. Note that TI needs not require the extra side information and the receiver only needs to know how to map the redundant constellations on the original one. Some modifications of TI have been proposed to obtain good performance including PAPR reduction and low complexity [31]. S.Janaththanan et.al [ 32] have proposed a novel gradient based approach for PAPR reduction using TR technique. The algorithm performs better but depends on the pilot locations.

The TI technique has been more problematic than the TR technique since the injected signal occupies the frequency band as the information bearing signals. Moreover, the alternative constellation points in TI technique have an increased energy and the implementation complexity increases for the computation the optimal translation vector.

\subsection{CodingTechniques}

Coding can also be used to reduce the PAPR. A recursive convolutional code, has been often used in OFDM system as a channel code. Yan Xin et al. [33] have proposed an integration of guided scrambling (GS) coding with SLM and PTS. The new GS-SLM and GS-PTS systems do not require transmission of side information and can be implemented resulting in good PAPR performance. Reed-Solomon and simplex codes has been proposed by Robert et al. [34] for PAR reduction in OFDM. An adaptive coding technique has been proposed by Zafar et al. [35] for reducing PAPR in COFDM to achieve reduction in PAPR as well as error correction capability. The adaptive approach has been adopted in order to reduce hardware for a slight increase in complexity. The coding technique Error Correction (EC) SLM using convolutional codes proposed by K.Khan et.al[36] has been studied. Guided polynomial scrambling technique gives the 
ISSN (Online): 1694-0814

www.IJCSI.org

most effective PAPR performance but slight degradation of BER.

\section{CONCLUSIONS}

In this paper some PAPR reduction techniques for multicarrier transmission have been discussed. Many techniques to reduce the PAPR have been proposed all of which have the potential to provide substantial reduction in PAPR at the cost of loss in data rate, transmit signal power increase, BER increase, computational complexity increase and so on. No specific PAPR reduction technique has been the best solution for all multicarrier transmission system. It has been suggested that the PAPR reduction technique should be carefully chosen according to various system requirements.

\section{References}

[1] S.H.Han and J.H.Lee, "An Overview of Peak-to-Average Power Ratio Reduction Techniques For Multicarrier Transmission," IEEE Wireless Communications,Vol.12,No.2, Apr.2005, pp.56-65.

[2] A. Ghassemi and T. Aaron Gulliver, "A low-Complexity PTS-Based Radix FFT Method for PAPR Reduction in OFDM Systems," IEEE Transactions on Signal Processing, Vol.56, No.3, March 2008, pp.11611166.

[3] R.S.Sheratt and O.Cadenas. J, "A Double Data Rate Architecture for OFDM Based Wireless Consumer Devices," IEEE Transactions on Consumer Electronics, Vol.56, No.1, February 2010, pp.23-26.

[4] S.H.Han and J.H.Lee, "PAPR Reduction of OFDM Signals using a Reduced Complexity PTS Technique," IEEE Signal Processing Letters, Vol.11, No.11, November 2004, pp.887-890.

[5] T.Jiang, W.Xiang, P.C.Richardson, J.Guo, and G. Zhu, "PAPR Reduction of OFDM Signals Using Partial Transmit Sequences with Low Computational Complexity," IEEE Transactions on Broadcasting, Vol.53, No.3, September 2007, pp.719-724.

[6] C.L.Wang, M.Y.Hsu, and Y.Wuynag,"A Low-Complexity Peak-toAverage Power Ratio Reduction Technique for OFDM systems,"Global Telecommunication Conference,2003 Globecom'03 IEEE, pp.23752379.

[7] Y.C.Cho, S.H.Han and J.H.Lee, "Selected Mapping Technique with Novel Phase Sequences for PAPR Reduction of an OFDM Signal," in Proc. of 5th IEEE VTC 2004-Fall, vol. 7, Sep. 2004, pp. 4781-4785.

[8] G.Hill and M.Faulkner, "Comparison of Low Complexity Clipping Algorithms for OFDM," IEEE International Symposium on Personal, Indoor and Mobile Radio communications, 2002,Vol..1, pp.227-231.

[9] H.Ochiai and H.Imai, "Performance Analysis of deliberately clipped OFDM signals," IEEE Trans. on communications, Vol.50, No.1, January 2002, pp.89-101.

[10] S.KYusof and N.Fisal,“ Coorelative Coding with Clipping and Filtering Technique in OFDM Systems," ICICS-PCM 2003, Singapore, IEEE 2003, pp.1456-1459.

[11] T.Jiang and Y.Wu, "An Overview: Peak-to-Average Power Ratio Reduction Techniques for OFDM Signals," IEEE Transactions on Broadcasting, Vol.54, No.2, June 2008, pp.257-268.

[12] D.Guel and J.Palicot, "FFT/IFFT Pair based Digital Filtering for the Transformation of Adding Signal PAPR Reduction Techniques in Tone Reservation Techniques," Fifth International Conference on Wireless and Mobile communications, (ICWMC 2009), August 2009.

[13] K.D.Rao and T.S.N.Murthy, "Analysis of Effects of Clipping and Filtering on the Performance of MB-OFDM UWB Signals," Proc. of the $200715^{\text {th }}$ International Conference on Digital Signal Processing (DSP 2007), IEEE, pp.559-562.

[14] S.Kimura,T.Nakamura,M.Saito and M.Okada,“ PAR reduction of OFDM signals based on deep clipping," ISCCSP 2008, Malta, 12-14 March 2008, IEEE, pp.911-916.

[15] D.Guel and J.Palicot,“ Analysis and Comparison of Clipping Techniques for OFDM Peak-to-Average Power Ratio Reduction," International Conference on Digital Signal Processing (DSP 2009), IEEE.

[16] X.Li and L.J.Cimini,“ Effects of Clipping and Filtering on the Performance of OFDM," IEEE Communication Letters, Vol.2, No.5, May 1998, pp.131-133.

[17] Q.Hua,R.Raich,G.T.Zhou,“ On the Benefits of Deliberately Introduced Baseband Nonlinearities in Communication Systems,", IEEE International Conference on Acoustics, Speech and Signal Processing (ICASSP'04), Vol.2, May 2004, pp.905-908.

[18] P.Boonsrimuang,E.Puttawong, H.Kobayashi and T.Paungma,“ PAPR Reduction Using Smooth Clipping in OFDM Systems," The 3rd Information and Computer Engineering Postgraduate Workshop 2003 (ICEP'2003), Jan2003, pp.158-161.

[19] T.Ya-fei, D.Rong-hua, Y.Xiao-an and T.Hai-wei,“ PAPR Reduction of OFDM Signals Using Modified Partial Transmit Sequences," $2^{\text {nd }}$ International Conference on Image and Signal Processing, 2009, CISP'09, pp.1-4

[20] J.C.Chen, "Partial Transmit Sequences for Peak-to-Average Power Ratio Reduction of OFDM Signals With the Cross-Entropy Method," IEEE Signal Processing Letters, Vol.16, No.6, June 2009, pp.545-548.

[21] L.Yang, R.S.Chen, Y.M.Siu, and K.K.Soo, "PAPR Reduction of an OFDM Signal by use of PTS with Low Computational Complexity," IEEE Trans. Broadcast., Vol.52, No.1, March 2006, pp.83-86.

[22] J.C.Chen, "Application of Quantum-Inspired Evolutionary Algorithm to Reduce PAPR of an OFDM signal Using Partial Transmit Sequences Technique,” IEEE Trans. On Broadcasting, Vol.56, No.1, March 2010, pp.110-113.

[23] A.Ghassemi and T.A. Gulliver, "PAPR Application of OFDM Using PTS and Error-Correcting Code Subblocking," IEEE Trans. On Wireless Communications, Vol.9, No.3, March 2010, pp.980-989.

[24] S.H.Han and J.H.Lee, "PAPR Reduction of OFDM Signals using a Reduced Complexity PTS Technique," IEEE Signal Processing Letters, Vol.11, No.11, Nov.2004, pp.887-890.

[25] H.Breiling,S.H.Muller-Weinfurtner,and J.B.Huber, "SLM Peak-Power Reduction without Explicit Side Information," IEEE Communication Letters, Vol.5, No.5, June 2001, pp.239-241.

[26] R.W.Bauml, R.F.H.Fisher and J.B.Huber, "Reducing the Peak-toAverage Power Ratio of Multicarrier Modulation by Selected Mapping," IEEE Electronic Letters, Vol.32, Oct. 1996, pp.2056-2057.

[27] A.D.S.Jayalath and C.Tellambura, "SLM and PTS Peak-Power Reduction of OFDM Signals without side information," IEEE Transactions on Wireless Communications, Vol.4, Sept.2005, pp.20062013.

[28] S.Y.Le Goff, Al-Samahi, S.S.B.K.Khoo, C.C.Tsimenidis and B.S.Sharif, "Selected Mapping without side information for PAPR Reduction in OFDM," IEEE Transactions on Wireless Communications, Vol.8, No.7, July. 2009, pp.3320-3325.

[29] L.Wang and Y.Cao, “ Improved SLM for PAPR Reduction in OFDM Systems," International Workshop on Intelligent Systems and Applications, 2009, ISA2009, pp.1-4.

[30] X.Gu, S.Baek, and S.Park,"PAPR Reduction of OFDM Signal Using an Efficient SLM Technique," ICACT 2010, Feb.2010, pp.324-328.

[31] J.Armstrong, "Peak-to-Average Reduction for OFDM by Repeated Clipping and Frequency Domain Filtering," IEEE Electronics Letters, Vol.38, No.5, May 2002, pp.246-247.

[32] S.Janaaththanan,C.Kasparis and B.G.Evans, "A Gradient Based Algoritm for PAPR Reduction of OFDM using Tone Reservation Technique,"Vehicular Technology Conference,2008,VTC Spring 2008, pp.2977-2980.

[33] Y.Xin and I.Fair," Peak-to-Average Power Ratio of an OFDM signal using Guided Scrambling Coding," Global Telecommunication conference,Globecom 2003,IEEE, Vol.4, pp.2390-2394. 
ISSN (Online): 1694-0814

www.IJCSI.org

[34] ] R.F.H.fischer and C.Siegl,"Reed-Solomon Solomon and Simplex Codes for Peak-to-Average Power Ratio reduction in OFDM,"IEEE trans.on Information Theory, Vol.55,No.4, Apr.2009, pp.1519-1528.

[35] Z.Q.Taha and X.Liu,"An Adaptive Coding Technique for PAPR Reduction," Global Telecommunication conference, Globecom 2007, IEEE , pp.376-380.

[36] K.Khan and S.A.Sheikh, "PAPR Reduction of OFDM Signals using Convolutional Codes," Proc. of 2009 IEEE Student Conf. on Research and Development, 16-18 Nov 2009, UPM Serdang, Malaysia, pp.26-28.

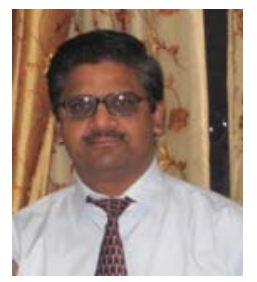

Pawan Sharma received the B.E. degree in 1995 and M.E. degree in 2004 from Delhi College of Engineering in Electronics and Communication. Worked in Bharati Vidyapeeth's College of Engineering, Amity Institute of Telecommunication. At present working in Bhagwan Parshuram Institute of Technology as an Assistant Professor in Electronics and Communication department.

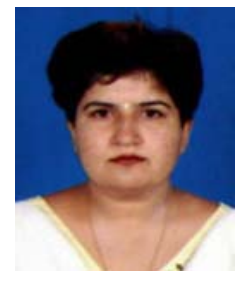

(Dr.) Seema Verma obtained her Ph.D degree from Banasthali University in 2003. She is working as Associate Professor [Electronics] at Banasthali University. She is an active research supervisor and has presented many papers in various international conferences. She has published many research papers in various journals of repute. Her research areas are Coding theory, TURBO Codes, Wireless sensor networks, Network Security \& VLSI Design. 